\title{
Violencia familiar durante el embarazo y su relación con el peso del recién nacido, Centro de Salud de Monterrey, Huaraz, 2012
}

Family violence during pregnancy and its relationship with the newborn weight at Monterrey Health Center, Huaraz, 2012

\author{
Yuliana De la Cruz Ramírez ${ }^{1}$, Augusto Olaza Maguiña ${ }^{1}$ Y \\ Nadezhda De la Cruz Ramírez ${ }^{2}$
}

\section{RESUMEN}

Este estudio determina la existencia de una relación entre la violencia familiar durante el embarazo y el peso de los recién nacidos en el Centro de Salud de Monterrey de Huaraz durante el año 2012. Se realizó una investigación retrospectiva, transversal, comparativa y correlacional en una muestra de 178 historias clínicas. Se usó una ficha de registro de datos. La información se procesó mediante el programa SPSS V20.0, utilizando las pruebas Chi cuadrado y diferencia de proporciones. Los resultados evidenciaron que la mayoría de gestantes sufrieron violencia psicológica $(58,1 \%)$; mientras que el $64,1 \%$ de los recién nacidos presentaron un peso adecuado al nacer, seguido de los neonatos con bajo peso $(35,9 \%)$. Se concluyó la existencia de una relación estadísticamente significativa, con un índice de confianza superior al 95\% entre la violencia familiar durante el embarazo y el peso de los recién nacidos; se evidenció también una diferencia estadísticamente significativa entre el peso de los neonatos según la violencia física, psicológica, económica o sexual de la cual fueron víctimas sus madres.

Palabras clave: violencia familiar; embarazo; peso; recién nacido.

\begin{abstract}
This research determines the existence of a relationship between family violence during pregnancy and weight of the newborns at the Monterrey Health Center of Huaraz in 2012, for which a retrospective, cross-sectional, comparative and correlational research was conducted in a sample of 178 medical records. A data collection document was used. The information was processed by means of SPSS V20.0 software, using Chi Square and difference of proportions tests. The results showed that the majority of pregnant women suffered psychological violence $(58,1 \%)$; while $64,1 \%$ of newborns had adequate weight, followed by underweight $(35,9 \%)$. It was concluded the existence of a statistically significant relationship, with an index confidence above $95 \%$, between the family violence during pregnancy and the weight of the newborns; demonstrating also a statistically significant difference between the weight of newborns according to the physical, psychological, economic or sexual violence that suffered their mothers.
\end{abstract}

Keywords: family violence; pregnancy; weight; newborn.

\footnotetext{
1 Universidad Nacional «Santiago Antúnez de Mayolo». Huaraz, Perú.

2 Hospital «Víctor Ramos Guardia». Huaraz, Perú.
} 


\section{ICHIKLLACHAW}

Kay musyapakuyqa rurakashqa imanawmi kastakunapa kikin wayinkunachaw warmikuna chichuyninchaw allqutsay kanqantam, hina llullu wamrakuna lasaynin yuriyanqanchaw kanqantam, Waras, Muntirriypa hampikuna wayinchaw, kay 2012 watachaw kashqanta, tsaypaqmi rurakashqa huk ritruspiktiba musyapakuyta, transbirsal qaqantsaywan kurilasiyunal nishqanwan, 178; tsaypaq huk rihistru huwichachaw rurakashqa. Kay llapan willakuykuna yaykunaq huk prugramaman SPSS V20.0, nishqanman, tsaypaq Chi chusku kuchuyuqta wanakashqa hina prupursiyun huklaayayninkunatam wanakashqa. Llapan yarqushqachaw rikakushqa imanawmi llapan chichushqakuna hipayarqan yuyay allqutsayninta $(58,1 \%)$; hukninkunaqa $64.1 \%$ tsayraq yuriyanqanchaw kaykaanaq allim lasayninkuna, tsayman qatin niwnatu huk ichik lasaywan (35,9\%). Uchuklla ushayninchaw istadistika alli kayninchaw tinkuynin kaptin huk indisi hatun markaynin kashqa 95\% kastapa allqutsayninwan chichuyninchaw, hinaman tsayraq yurikushqakunapa lasaynin; tsaypitanam tsay rikakashqa istadistikaminti huklaayaynin alli kashqa tsayraq yurikushqakunapa lasayninwan, huwisika allqutsayninraykur, sikuluhika, ikunumika aw pinqakuy, tsayraykurmi mamankuna allqutsashqa hina ashashqa kayaanaq.

Pushaq shimikuna: kastakunapa allqutsaynin; chichuy; lasay; tsayraq yurishqa.

\section{INTRODUCCIÓN}

La presente investigación aborda la problemática sobre la violencia familiar durante el embarazo, cuyos casos fueron atendidos durante el año 2012 en el Centro de Salud de Monterrey, establecimiento de salud de categoría I-4 de nivel de atención, ubicado en el Centro Poblado del mismo nombre, perteneciente al distrito de Independencia, provincia de Huaraz, región Áncash. La población que se atiende en dicho establecimiento procede en un $40 \%$ del área rural, con un nivel educativo promedio de secundaria incompleta, quechuahablantes en más del $50 \%$ y un promedio de ingreso económico mensual apenas superior al sueldo mínimo vital (Universidad del Pacífico, 2011). En este contexto, al igual que en otras regiones del Perú, la violencia hacia la mujer es un hecho frecuente y una causa significativa de morbilidad y mortalidad femenina, pues estimaciones del Banco Mundial indican que la violencia dirigida a la mujer hace que se pierda uno de cada cinco días de vida saludable en edad reproductiva (Valdez y Sanín, 2006).

En el Congreso Internacional «Violencia de Género» llevado a cabo en Santiago de Compostela, España (2004), se concluyó que en países con realidades similares a las descritas anteriormente, en el 90\% de los casos el abusador es el conviviente de las gestantes, quienes en un $25 \%$ a $50 \%$ sufren golpes en el abdomen, lo cual tiene consecuencias importantes en la salud tanto de ellas como del producto del embarazo (Heise 2004, 25), destacándose que dicha violencia familiar se relaciona con el aumento de estrés que siente el esposo o conviviente con respecto al parto inminente, el cual se manifiesta como una frustración que dirige contra la madre y su niño no nacido (World Health Organization, 2011).

La violencia familiar durante el embarazo es una agresión que pone en peligro no solo una sino dos vidas; pues pone a la mujer embarazada y al feto en situación de riesgo para su salud física y mental, constituyéndose en un factor que influye para un recién 
nacido de bajo peso, es decir que la violencia tiene consecuencias sobre el producto y configura lo que se conoce como abuso prenatal (Mosesson, Rosemberg y Lapidus, 2010).

Otro estudio realizado por Alponte en México (2011), en el Estado de Morelos, notificó una prevalencia de violencia en el embarazo de 33,5\% en mujeres usuarias de un hospital público, las mismas que tuvieron cuatro veces más riesgo de dar a luz productos de bajo peso al nacer que las que no sufrían de violencia, sumado a que los recién nacidos eran 40 veces más propensos de morir en el primer año de vida.

Como lo demuestran las cifras, la violencia doméstica ataca a todos los miembros vulnerables de la familia, pero principalmente a las mujeres de todas las edades. Los hallazgos muestran que las mujeres del área rural peruana tienen un índice alto de violencia y maltrato, el cual bordea cifras entre 25 y 62\% (Rachana et al., 2002).

De acuerdo con un estudio llevado a cabo por la organización Flora Tristán (2011), el $51 \%$ de las mujeres en Lima y el 69\% en Cusco han sido golpeadas al menos una vez en su vida por su pareja; además que del total de mujeres alguna vez embarazadas, el 14,8\% reportaron violencia física en Canta (Lima) y 27,6\% en la provincia cusqueña de Chumbivilcas. Según una investigación realizada por el Ministerio de la Mujer y Poblaciones Vulnerables del Perú en el 2008, 88,2\% de las mujeres en Lima y Callao conocen personalmente a otras mujeres que son víctimas de violencia familiar, mientras que el 29\% de mujeres maltratadas en las provincias del Perú no piden ayuda. En una encuesta realizada sobre los motivos para no pedir ayuda, se destaca el considerar a la violencia como algo normal o no serio, el temor a las consecuencias, la pérdida de sus hijos, la vergüenza para la familia, el que nadie les creyera o que contarlo no ayudaría.

Por la importancia del tema y en ausencia de estudios similares a nivel local, se realizó la presente investigación con el objetivo de determinar la existencia de una relación entre la violencia familiar durante el embarazo y el peso de los recién nacidos en el Centro de Salud de Monterrey de Huaraz durante el año 2012. Los objetivos específicos fueron: Determinar la prevalencia de los tipos de violencia familiar durante el embarazo; identificar la prevalencia del buen y bajo peso del recién nacido de mujeres que sufrieron violencia familiar durante el embarazo; precisar las características sociodemográficas de las mujeres embarazadas y su relación con el peso del recién nacido; y, comparar la prevalencia de la violencia familiar durante el embarazo según el peso del recién nacido.

\section{MATERIALES Y MÉTODOS}

Se llevó a cabo un estudio retrospectivo, transversal, comparativo y correlacional (Hernández, Fernández y Baptista, 2010; Canales, Alvarado y Pineda, 2006). La población estuvo conformada por todas las gestantes que sufrieron violencia familiar durante el embarazo y cuya atención de parto se realizó en el servicio de Gíneco-Obstetricia del Centro de Salud de Monterrey en Huaraz, las cuales fueron atendidas durante el año 2012, con un total de 220 gestantes y sus recién nacidos en la misma proporción. Se usó el muestreo probabilístico (ob. cit.), para lo cual se determinó el tamaño de la muestra, mediante la aplicación de la fórmula respectiva (Hopkins, 2008), obteniendo una muestra de 89 pacientes en cada grupo de gestantes víctimas de violencia familiar 
durante el embarazo con recién nacidos de buen y bajo peso (total 178 gestantes).

La información se obtuvo usando como instrumento una ficha de registro de datos a partir de historias clínicas de gestantes que contenían fichas de tamizaje de violencia familiar, respetándose en todo momento las normas éticas internacionales. La validez del instrumento se realizó mediante juicio de expertos, cuya prueba de concordancia de Kendall, demostró su validez con un nivel de significancia del 0,0001. Asimismo, luego de la aplicación de una prueba piloto, se procedió al cálculo de la confiabilidad del instrumento mediante el Coeficiente alfa de Cronbach, obteniéndose como resultado un instrumento de investigación confiable $(0,854)$.

Para el procesamiento de la información se usó el programa estadístico Statistics Program for Social Science V20.0. (IBM Corporation, 2012), utilizándose como instrumento de análisis las tablas y gráficos de frecuencia, así como las pruebas Chi cuadrado y diferencia de proporciones $(\alpha=0,05)$ (Freund, 2009).

\section{RESULTADOS}

Prevalencia de los tipos de violencia familiar durante el embarazo:

Tabla 1. Prevalencia de la violencia familiar durante el embarazo

\begin{tabular}{lcc}
\hline \multirow{2}{*}{$\begin{array}{c}\text { Tipos de violencia familiar durante el } \\
\text { embarazo }\end{array}$} & \multicolumn{2}{c}{ Prevalencia de los tipos de violencia familiar } \\
\cline { 2 - 3 } & Número de casos & Prevalencia \\
\hline Violencia física & 34 & $15,5 \%$ \\
Violencia psicológica & 128 & $58,1 \%$ \\
Violencia económica & 35 & $15,9 \%$ \\
Violencia sexual & 23 & $10,5 \%$ \\
Total & 220 & $100 \%$ \\
\hline
\end{tabular}

En la tabla 1 se evidencia que el 58,1\% del total de casos de violencia familiar durante el embarazo (220) registrados en el Centro de Salud Monterrey de Huaraz, correspondió a mujeres víctimas de violencia psicológica, observándose un 15,5\% de atenciones por violencia física.

Prevalencia del peso adecuado y bajo peso del recién nacido en mujeres que sufrieron violencia familiar durante el embarazo:

Tabla 2. Prevalencia del peso del recién nacido en mujeres que sufrieron violencia familiar durante el embarazo

\begin{tabular}{lcc}
\hline \multirow{2}{*}{ Peso del recién nacido } & \multicolumn{2}{c}{ Prevalencia } \\
\cline { 2 - 3 } & 141 & Púmero de casos \\
\hline Peso adecuado & 79 & $64,1 \%$ \\
Bajo peso & 220 & $35,9 \%$ \\
Total & $100 \%$ \\
\hline
\end{tabular}


En la tabla 2 se observa que la prevalencia del peso adecuado en los recién nacidos cuyas madres fueron víctimas de violencia familiar durante el embarazo (220), fue del $64,1 \%$, seguido por el $35,9 \%$ que correspondió a los casos de recién nacidos con bajo peso.

Características sociodemográficas de las mujeres embarazadas y su relación con el peso del recién nacido:

Tabla 3. Características sociales de las mujeres embarazadas y su relación con el peso del recién nacido

\begin{tabular}{|c|c|c|c|c|c|c|c|}
\hline \multirow{3}{*}{ Características sociales } & \multicolumn{4}{|c|}{ Peso del recién nacido } & \multirow{2}{*}{\multicolumn{2}{|c|}{ Total }} & \multirow{3}{*}{$\begin{array}{l}\text { Resultado del } \\
\text { Chi cuadrado }\end{array}$} \\
\hline & \multicolumn{2}{|c|}{ Peso adecuado } & \multirow{2}{*}{$\begin{array}{l}\text { Bajo } \\
\mathrm{N}^{\circ}\end{array}$} & \multirow{2}{*}{$\frac{\text { peso }}{\%}$} & & & \\
\hline & $\mathrm{N}^{\circ}$ & $\%$ & & & $\mathrm{~N}^{\circ}$ & $\%$ & \\
\hline \multicolumn{8}{|l|}{ Procedencia: } \\
\hline Urbano & 63 & 35,4 & 49 & 27,5 & 112 & 62,9 & $\mathrm{X}_{\mathrm{c}}^{2}=4,720$ \\
\hline Rural & 26 & 14,6 & 40 & 22,5 & 66 & 37,1 & $\mathrm{p}=0,030$ \\
\hline Total & 89 & 50 & 89 & 50 & 178 & 100 & \\
\hline \multicolumn{8}{|l|}{ Ocupación: } \\
\hline Ama de casa & 57 & 32 & 62 & 34,9 & 119 & 66,9 & \\
\hline Estudiante & 11 & 6,2 & 15 & 8,4 & 26 & 14,6 & $\mathrm{X}_{\mathrm{c}}^{2}=3,280$ \\
\hline $\begin{array}{l}\text { Trabajadora dependiente o } \\
\text { independiente }\end{array}$ & 21 & 11,8 & 12 & 6,7 & 33 & 18,5 & $\mathrm{p}=0,194$ \\
\hline Total & 89 & 50 & 89 & 50 & 178 & 100 & \\
\hline \multicolumn{8}{|l|}{ Ingreso económico: } \\
\hline Percibe ingreso propio & 21 & 11,8 & 14 & 7,9 & 35 & 19,7 & $\mathrm{X}_{\mathrm{c}}^{2}=1,743$ \\
\hline No percibe ingreso propio & 68 & 38,2 & 75 & 42,1 & 143 & 80,3 & $\mathrm{p}=0,187$ \\
\hline Total & 89 & 50 & 89 & 50 & 178 & 100 & \\
\hline \multicolumn{8}{|l|}{ Hábitos nocivos: } \\
\hline Sí & 1 & 0,6 & 2 & 1,1 & 3 & 1,7 & $\mathrm{X}_{\mathrm{c}}^{2}=0,339$ \\
\hline No & 88 & 49,4 & 87 & 48,9 & 175 & 98,3 & $\mathrm{p}=0,560$ \\
\hline Total & 89 & 50 & 89 & 50 & 178 & 100 & \\
\hline
\end{tabular}

En la tabla 3 se observa que la mayoría de mujeres que sufrieron violencia durante su gestación fueron procedentes del área urbana (62,9\%), desempeñándose como amas de casa $(66,9 \%)$, sin un ingreso económico propio $(80,3 \%)$ y sin la presencia de hábitos nocivos $(98,3 \%)$. Asimismo, se evidenció una relación estadísticamente significativa entre la procedencia y el peso del recién nacido ( $\mathrm{p}<0,05)$; no observándose lo mismo con la ocupación, ingreso económico y hábitos nocivos de las gestantes $(\mathrm{p}>0,05)$. 
Tabla 4. Características demográficas de las mujeres embarazadas y su relación con el peso del recién nacido

\begin{tabular}{|c|c|c|c|c|c|c|c|}
\hline \multirow{3}{*}{$\begin{array}{l}\text { Características } \\
\text { demográficas }\end{array}$} & \multicolumn{4}{|c|}{ Peso del recién nacido } & & & \multirow{3}{*}{$\begin{array}{l}\text { Resultado } \\
\text { del Chi } \\
\text { cuadrado }\end{array}$} \\
\hline & \multicolumn{2}{|c|}{ Peso adecuado } & \multirow{2}{*}{$\frac{\text { Bajo }}{\mathrm{N}^{\circ}}$} & \multirow{2}{*}{$\frac{\text { peso }}{\%}$} & \multicolumn{2}{|c|}{ Total } & \\
\hline & $\mathrm{N}^{\circ}$ & $\%$ & & & $\mathrm{~N}^{\circ}$ & $\%$ & \\
\hline \multicolumn{8}{|l|}{ Edad materna: } \\
\hline $15-20$ años & 17 & 9,6 & 44 & 24,7 & 61 & 34,3 & $\mathrm{X}_{\mathrm{c}}^{2}=18,182$ \\
\hline $21-35$ años & 72 & 40,4 & 45 & 25,3 & 117 & 65,7 & $\mathrm{p}=0,000$ \\
\hline Total & 89 & 50 & 89 & 50 & 178 & 100 & \\
\hline \multicolumn{8}{|l|}{ Estado civil: } \\
\hline Soltera & 2 & 1,1 & 12 & 6,7 & 14 & 7,8 & \\
\hline Casada & 11 & 6,2 & 14 & 7,9 & 25 & 14,1 & $\mathrm{X}_{\mathrm{c}}^{2}=12,385$ \\
\hline Conviviente & 76 & 42,7 & 60 & 33,7 & 136 & 76,4 & $p=0,006$ \\
\hline Viuda & 0 & 0 & 3 & 1,7 & 3 & 1,7 & \\
\hline Total & 89 & 50 & 89 & 50 & 178 & 100 & \\
\hline \multicolumn{8}{|l|}{ Grado de instrucción: } \\
\hline Analfabeta & 1 & 0,6 & 8 & 4,5 & 9 & 5,1 & \\
\hline Primaria & 2 & 1,1 & 22 & 12,4 & 24 & 13,5 & $\mathrm{X}_{\mathrm{c}}^{2}=27,139$ \\
\hline Secundaria & 73 & 41 & 50 & 28 & 123 & 69 & $\mathrm{p}=0,000$ \\
\hline Superior & 13 & 7,3 & 9 & 5,1 & 22 & 12,4 & \\
\hline Total & 89 & 50 & 89 & 50 & 178 & 100 & \\
\hline
\end{tabular}

En la tabla 4, se da a conocer que los mayores porcentajes de violencia familiar durante el embarazo correspondieron a gestantes cuya edad estuvo comprendida entre los 21 y 35 años (65,7\%), convivientes $(76,4 \%)$ y con estudios del nivel secundario $(69 \%)$, evidenciándose una relación estadísticamente significativa de estas variables con el peso del recién nacido $(\mathrm{p}<0,05)$.

Relación entre la violencia familiar durante el embarazo y el peso del recién nacido.

Tabla 5. Relación entre la violencia familiar durante el embarazo y el peso del recién nacido

\begin{tabular}{|c|c|c|c|c|c|c|}
\hline \multirow{3}{*}{ Tipos de violencia familiar } & \multicolumn{4}{|c|}{ Peso del recién nacido } & \multirow{2}{*}{\multicolumn{2}{|c|}{ Total }} \\
\hline & \multicolumn{2}{|c|}{ Peso adecuado } & \multicolumn{2}{|c|}{ Bajo peso } & & \\
\hline & $\mathrm{N}^{\circ}$ & $\%$ & $\mathrm{~N}^{\circ}$ & $\%$ & $\mathrm{~N}^{\circ}$ & $\%$ \\
\hline Violencia física & 2 & 0,9 & 32 & 14,6 & 34 & 15,5 \\
\hline Violencia psicológica & 102 & 46,4 & 26 & 11,7 & 128 & 58,1 \\
\hline Violencia económica & 30 & 13,6 & 5 & 2,3 & 35 & 15,9 \\
\hline Violencia sexual & 7 & 3,2 & 16 & 7,3 & 23 & 10,5 \\
\hline \multirow[t]{2}{*}{ Total } & 141 & 64,1 & 79 & 35,9 & 220 & 100 \\
\hline & \multicolumn{2}{|c|}{$X_{c}^{2}=82,016$} & \multicolumn{2}{|c|}{$Z_{c}=-6,965$} & \multicolumn{2}{|c|}{$p=0,000$} \\
\hline
\end{tabular}


La tabla 5 constituye la respuesta directa a la hipótesis y objetivo general de la investigación, evidenciándose que el 14,6\% de casos correspondió a mujeres víctimas de violencia física y que a la vez tuvieron un recién nacido de bajo peso. Asimismo, luego del análisis estadístico se demostró una relación estadísticamente significativa entre el tipo de violencia familiar y el peso de los recién nacidos; así como también se evidenció una diferencia significativa entre el peso de los neonatos de acuerdo al tipo de violencia del cual fueron víctimas sus madres $(\mathrm{p}<0,05)$, especialmente en la violencia física ( $0,9 \%$ vs. $14,6 \%)$; con lo cual se dio respuesta al cuarto objetivo específico orientado a comparar la prevalencia de la violencia familiar durante el embarazo según el peso del recién nacido.

\section{DISCUSIÓN}

Los resultados de la presente investigación, con respecto al primer objetivo específico orientado a determinar la prevalencia de los tipos de violencia familiar durante el embarazo, fueron dados a conocer en la tabla 1, en la cual se observó que el 58,1\% del total de casos de violencia familiar durante el embarazo (220) registrados en el Centro de Salud Monterrey de Huaraz, correspondió a mujeres víctimas de violencia psicológica, hallazgo que guarda relación con el estudio realizado por Cuamatzi, García y Cruz (2004), quienes evidenciaron que la mujer maltratada desarrolla un cuadro de estrés permanente que se asocia con depresión, angustia, baja autoestima, aislamiento, suicidio y homicidio. Las consecuencias psicológicas a largo plazo de la violencia doméstica durante el embarazo tienen un impacto en el peso del producto al nacer, lo cual lleva consigo un efecto perjudicial severo en el desarrollo psicológico del niño, quien probablemente será testigo de violencia doméstica después de su nacimiento. Asimismo, Castro (2012) encontró como más frecuente el abuso psicológico, caracterizado por humillaciones verbales, gritos, groserías, abandono y amenaza de maltrato, el mismo que se presentó en el $46 \%$ de casos; mientras que el abuso físico y sexual se dio en el $40 \%$ y $14 \%$, respectivamente; cifras mayores a las reportadas en la presente investigación $(15,5 \%$ y $10,5 \%$, respectivamente).

Con respecto a la identificación de la prevalencia del buen y bajo peso del recién nacido de mujeres que sufrieron violencia familiar durante el embarazo, el cual se constituyó en el segundo objetivo específico de la presente investigación (tabla 2), se evidenció que el $64,1 \%$ tuvieron adecuado peso al nacer, seguido por el $35,9 \%$ con bajo peso, condición que aunque no fue mayoritaria, sí resultó importante. Una conclusión similar fue dada a conocer por Núñez, Monge, Gríos, Elizondo y Rojas (2009), quienes pusieron de manifiesto que los niños de madres que sufrieron actos de violencia pesaron en promedio 449,4 g menos $(\mathrm{p}<0,001)$ que los de las mujeres que no habían estado expuestas a actos de violencia. Las primeras presentaron un riesgo tres veces mayor de tener hijos con bajo peso al nacer que las segundas (IC 95\%: 1,39 a 8,10). Las variables que se asociaron más estrechamente con el bajo peso al nacer fueron la violencia sufrida por la madre (asociación directa) y el aumento de peso de la madre durante la gestación (relación inversa).

En las tablas 3 y 4 se pone de manifiesto la respuesta al tercer objetivo del presente estudio, orientado a precisar las características sociodemográficas de las mujeres embarazadas y su relación con el peso del recién nacido, concluyéndose que a excepción de 
la ocupación, ingreso económico y hábitos nocivos de las gestantes, el resto de características sociodemográficas como la procedencia, edad materna, estado civil y grado de instrucción sí guardan una relación significativa con el peso al nacer; hallazgos similares a los reportados por Cuamatzi, García y Cruz (2004), quienes manifestan que la edad media fue de 13 a 16 años, con un nivel de escolaridad de primaria completa (61,5\%). La mayoría de las mujeres eran amas de casa, solo 14\% tenía algún tipo de empleo y de estas, la mitad eran trabajadoras domésticas, el ingreso familiar mensual fluctuaba entre $\$ 16,00$ y $\$ 132,00$; con respecto al estado civil, el $82,7 \%$ era casada o conviviente; de las cuales $47 \%$ estaban casadas y las restantes vivían en unión libre. El $17,3 \%$ eran madres solteras. El 33,5\% fue violentada por sus parejas; $5,4 \%$ cuando el conviviente se enteró del embarazo, una fue violada representando el 3\% de casos. La mayoría de las gestantes adolescentes declaró experimentar diferentes tipos de abuso por parte de la pareja. El uso de drogas resultó bajo, ya que solo en 7 de los casos la pareja violenta estaba bajo los efectos de alguna droga en el momento de la agresión.

En la tabla 5 no solo se da a conocer los resultados con respecto al cuarto objetivo específico (comparar la prevalencia de la violencia familiar durante el embarazo según el peso del recién nacido), sino que también se expone los hallazgos con respecto a la hipótesis y objetivo general de la presente investigación. Se da a conocer los resultados de la relación entre la violencia familiar durante el embarazo y el peso del recién nacido en el Centro de Salud de Monterrey de Huaraz durante el año 2012; evidenciándose que el 14,6\% de casos correspondió a mujeres víctimas de violencia física y que a la vez tuvieron un recién nacido de bajo peso; demostrándose una relación estadísticamente significativa entre las variables del estudio, además de una diferencia significativa entre el peso de los neonatos de acuerdo al tipo de violencia del cual fueron víctimas sus madres, $(\mathrm{p}<0,05)$.

$\mathrm{Al}$ respecto, estos resultados guardan relación con lo hallado por diversos investigadores, como Oyague (2002) en la provincia de Huaraz, quien enfatizó la alta prevalencia del abuso a mujeres embarazadas por sus parejas o ex parejas; los efectos nocivos de la violencia (en la salud física, reproductiva y mental) y el impacto de la violencia en el peso del recién nacido, conclusión ratificada también por Rodríguez (2010), Cuevas, Blanco, Juárez, Palma y Valdez (2006).

Finalmente, la reflexión epistemológica sobre los resultados antes mencionados, permite señalar que el abordaje de la violencia familiar durante el embarazo en el Centro de Salud de Monterrey de Huaraz, solo se circunscribe a un modelo médico, inapropiado para abordar los fenómenos psicosociales, siendo importante la aplicación de un enfoque con perspectiva de género, basado en la atención inter y multidisciplinaria de los diferentes niveles de realidad en los que se desarrolla el fenómeno de la violencia.

\section{CONCLUSIONES}

Existe una relación estadísticamente significativa entre el tipo de violencia familiar y el peso de los recién nacidos en el Centro de Salud de Monterrey de Huaraz; evidenciándose que el 14,6\% de casos correspondió a mujeres víctimas de violencia física y que a la vez tuvieron un recién nacido de bajo peso. 
El tipo de violencia familiar que mostró mayor prevalencia fue la violencia psicológica con el $58,1 \%$, mientras que la violencia sexual presentó menor proporción de casos con el 10,5\%.

Con respecto a la prevalencia del peso de recién nacidos en mujeres que sufrieron violencia familiar durante el embarazo, se concluye que dicha prevalencia fue mayor en el grupo de neonatos con peso adecuado $(64,1 \%)$, seguido de aquellos con bajo peso $(35,9 \%)$.

Las características sociodemográficas que evidenciaron una relación estadísticamente significativa con el peso del recién nacido fueron la edad materna, procedencia, estado civil y grado de instrucción; no observándose lo mismo con la ocupación, ingreso económico y hábitos nocivos de las gestantes.

Se concluye una diferencia estadísticamente significativa entre el peso de los neonatos de acuerdo al tipo de violencia del cual fueron víctimas sus madres, evidenciándose una mayor diferencia con respecto a los casos de violencia física entre los recién nacidos con peso adecuado $(0,9 \%)$ y bajo peso $(14,6 \%)$.

\section{AGRADECIMIENTOS}

Al personal asistencial del Centro de Salud de Monterrey de Huaraz; por su colaboración y entusiasmo demostrado durante el desarrollo del presente estudio.

\section{REFERENCIAS BIBLIOGRÁFICAS}

Alponte, Juan. 2011. La violencia y la mujer en México. México D.F.: El Ateneo.

Canales, Francisca; Alvarado, Eva y Pineda, Elia. 2006. Metodología de la investigación. Manual para el desarrollo de personal de salud. México D.F.: Limusa.

Castro, Roberto. 2012. Estudio nacional sobre las fuentes, origenes y factores que producen y reproducen la violencia contra las mujeres. México D.F.: Comisión Nacional para Prevenir y Erradicar la Violencia contra las Mujeres.

Cuamatzi, María; García, Guadalupe y Cruz, Cruz. 2004. Repercusión en la salud de las madres adolescentes que viven en situación de violencia familiar en la comunidad de Nezabualcóyotl. Salud Pública México. 46(1). 12-7.

Cuevas, Sofía y otros. 2006. Violencia y embarazo en usuarias del sector salud en estados de alta marginación en México. Salud Pública México. 48(2). 239-249.

Freund, Jhon. 2009. Estadística elemental. México D.F.: Prentice-Hall Hispanoamericana.

Heise, Louis. 2004. Violence against women, the burden on health. Washington DC: Banco Mundial/OPS.

Hernández, Roberto; Fernández, Carlos y Baptista, Pilar. 2010. Metodología de la investigación. México D.F.: McGraw-Hill.

Hopkins, Billy. 2008. Estadística básica para las ciencias sociales y del comportamiento. México D.F.: Prentice-Hall Hispanoamericana. 
IBM Corporation. 2012. Statistics program for Social Science V20.0 [CD-ROM]. New York: IBM Corporation.

Ministerio de la Mujer y Poblaciones Vulnerables. 2008. Violencia familiar. Lima: MIMP.

Mosesson, Bertha; Rosemberg, Kenneth y Lapidus, Jodi. 2010. Intimate partner violence during pregnancy. Oregon: OPS.

Núñez, H. y otros. 2009. La violencia física, psicológica, emocional y sexual durante el embarazo: riesgo reproductivo predictor de bajo peso al nacer en Costa Rica. Revista Panamericana de Salud Pública. 14(2). 75-83.

ONG Flora Tristán. 2011. Violencia contra la Mujer: Feminicidio en el Perú. Lima: ONG Centro de la Mujer Peruana Flora Tristán.

Oyague, María. 2002. El castigo físico y psicológico: una pauta que queremos cambiar. Huaraz: Centro de Documentación (CENDOC), Centro de Estudios Sociales y Publicaciones Ancash.

Rachana, C. y otros. 2002. Prevalence and complications of physical violence during pregnancy. Eur J Obstet Gynecol Reprod Biol. 103(1). 26-29.

Rodríguez, Yolanda. 2010. Abordaje del método de historia de vida en el estudio de la violencia contra la mijer. In Crescendo Revista Científica de la Universidad Católica Los Ángeles de Chimbote. 1(1). 9-20.

Universidad del Pacífico. 2011. Estudio de Linea de Base 2011 de condiciones de vida y pobreza del distrito de Independencia (Huaraz). Lima: Universidad del Pacífico.

Valdez, Santiago, y Luis Sanín. 2006. La violencia doméstica durante el embarazo y su relación con el peso al nacer. México D.F.: El Ateneo.

World Health Organization. 2011. World report on violence and health. Washington D.C.: WHO.

Fecha de recepción: 19 de enero de 2015

Fecha de aceptación: 28 de marzo de 2015

\section{Correspondencia}

Yuliana De la Cruz Ramírez

yulianadr2@hotmail.com 